\title{
Efficiency of Wireless Power transfer with a Multi-sine Source Optimized for the Propagation Channel
}

\author{
$1^{\text {st }}$ Regis Rousseau \\ CITI Lab \\ Univ Lyon, INSA Lyon, Inria \\ F-69621 Villeurbanne, France \\ regis.rousseau@insa-lyon.fr
}

\author{
$2^{\text {nd }}$ Guillaume Villemaud \\ CITI Lab \\ Univ Lyon, INSA Lyon, Inria \\ F-69621 Villeurbanne, France \\ guillaume.villemaud@insa-lyon.fr
}

\author{
$3^{\text {nd }}$ Florin Hutu \\ CITI Lab \\ Univ Lyon, INSA Lyon, Inria \\ F-69621 Villeurbanne, France \\ florin-doru.hutu@insa-lyon.fr
}

\begin{abstract}
In this paper, the authors present an original solution to maximize the DC power collected in the case of a wireless power transfer (WPT) scenario. Using state-space model representation, the WPT System is considered as a feedback approach in order to maximize the amount of harvested energy. To do this, a global simulation is performed to show the importance of taking into account the propagation channel and the rectifier circuit aspects in the case of optimizing the waveform to increase the harvested energy. By using an optimized multisine signal with zero phase as the excitation, taking into account the characteristics of the channel and the physical contributions of the rectifier, we managed to obtain better output DC values compared to a single tone source or a multi-sine signal without optimization, with the same average power input.
\end{abstract}

Index Terms-Propagation channel, Waveform optimization, Multi-tone signal, Wireless power transmission, Energy harvesting.

\section{INTRODUCTION}

Maximizing DC power in an energy harvesting circuit using a multi-sine excitation has been proposed to obtain significant performance benefits compared to a continuous wave excitation since several years. For example, in a use case of passive RFID, the use of a multi-sine signal as an RF excitation source allows a gain of $10.4 \mathrm{~dB}$ for a 4 -subcarrier power-optimized waveform compared to the use a continuous wave, for the same average power [1]. If we consider a conventional envelope detector circuit consisting of a single rectifying diode, the use of a multi-sine signal as a source of excitation for this type of rectifier has also been theoretically demonstrated in [2], and this technique is further validated by simulation and measurements. The simulation results show, for an 8-tone signal at $5.8 \mathrm{GHz}$ for the first tone frequency, a gain of $9 \mathrm{~dB}$ regarding to a single tone case.

Since the main objective is of course to significantly increase the efficiency of the rectifier, efforts have been made on the rectifier excited by this multi-sine signal, for example to find the optimum load resistance according to the PAPR of the input signal [3].

Thus, many improvements from the circuit point of view have shown very encouraging prospects, even further via the use of high PAPR signals, for instance a multi-sine signal. But, in these works, simulations or experiments do not take into account the communication channel.

Other works show that this performance can also be improved by optimizing the amplitude of each sine of this multitone signal. Knowing the behavior of the propagation channel, an algorithm based on a sequential convex optimization has been developed [4] and has shown the importance of accounting for the channel state information and the nonlinearity of the rectifier in order to increase the collected DC power. A prototype has been designed [5] and shows significant performance increase with an adaptive 8-tone source.

In this study case and as part of an implementation of the waveform optimization algorithm, the rectifier is modeled in its smallest form i.e. the Taylor expansion of the nonlinear current equation of the Schottky diode (Shockley ideal diode equation). The parasitic part of the diode, time constant of the R-C cell and the imperfection of the impedance matching network are neglected.

In this paper, we develop and simulate a complete link taking into account the system as a whole. Section II presents a global view of WPT systems as a closed-loop. Section III gives the setup of the system architecture and section IV provides the simulation results in various conditions. Conclusions and future directions of this work are given in section V.

\section{WPT SYSTEM MODELED AS A CLOSED LOOP}

In [6], we introduced an original method of WPT system modeling by using state-space model representation. Each element of a WPT system (i.e. propagation channel and linear and nonlinear parts of rectifier) is expressed in the form of a similar state-space model, shown in Fig. 1.

At this stage of the project, the system is fully simulated under Keysights ADS software and Matlab. Because of the very low power level at the rectifier's antenna, the topology of the rectenna is the basic structure of a series-mounted diode. Transmitted and received antenna impedances are fixed to $50 \Omega$. Passive components of the impedance matching network and load resistance are optimized by a global circuit 


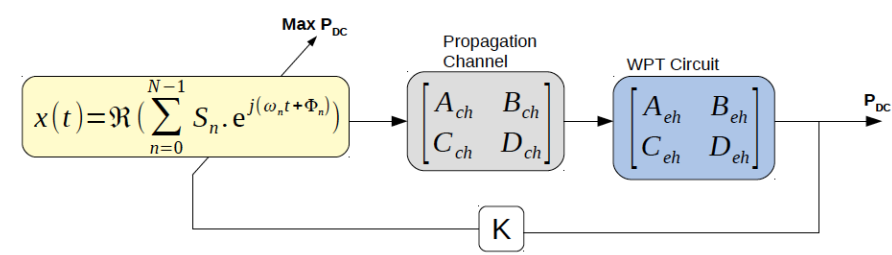

Fig. 1. A WPT system model. The propagation channel and the rectifying circuit are modeled by their respective state-space models.

optimization technique based on an $S_{11}$ parameter goal.

The state-space models of linear parts, impedance matching network and parasitic elements of the Schottky diode plastic package (SOT-23) of the rectifier are built from the vector fitting algorithm based on the S-parameters of each linear part.

To acquire the channel state estimation, the pilot-based channel estimation technique is used through the simulated fading channel and a Multivariable Output-Error State-space algorithm under the Matlab software is used to build the corresponding state-space model.

Then, the Schottky diode is considered in the form of a state-space model by the nodal DK method [7].

Once the system is fully modeled as state-space models, this problem could be formulated as a convex optimization.

\section{Simulation Model of the WPT System}

In order to know the gain obtained by optimizing the amplitudes of the input multi-sine signal of this closed-loop system, a first simulation on Keysights ADS software has been performed with realistic elements of a single diode rectifier and a multi-path channel.

\section{A. Rectifier Design}

The single diode rectifier circuit is designed by using a Skyworks SMS7630 Schottky diode. The rectifier input is matched to $50 \Omega$ by two L-matching networks (6-order LC bandpass filter). It guarantees an input reflection coefficient between $-20 \mathrm{~dB}$ and $-26 \mathrm{~dB}$ for an input signal of $2.45 \mathrm{GHz}$ having a $20 \mathrm{MHz}$ bandwidth and a $-40 \mathrm{dBm}$ input power level. The load resistance is optimized and its value is 15 $\mathrm{k} \Omega$. Tab. I gives some reference performance values of this proposed rectifier for a continuous wave (1-tone).

TABLE I

DC Voltage and Efficiency Performance Results

\begin{tabular}{|c|c|c|}
\hline Input & \multicolumn{2}{|c|}{ Output Values } \\
\hline Power (dBm) & DC Voltage $(\boldsymbol{m}$ V) & Efficiency (\%) \\
\hline-40 & 5 & 1.5 \\
\hline-30 & 40 & 11 \\
\hline-20 & 177 & 30 \\
\hline-10 & 500 & 48 \\
\hline
\end{tabular}

Recently, in [8], an experimental rectifier is presented with a single diode rectifier topology with a Broadcom HSMS2850 mounted in shunt. The measured peak efficiencies are respectively $4.3 \%, 24.3 \%, 48.5 \%$ with an input power level of $30,-20,-10 \mathrm{dBm}$ at the frequency of $2.472 \mathrm{GHz}$. So, the performance of the proposed rectifier gives relatively similar results and agrees with the expected performance of current rectifiers.

\section{B. Propagation Channel}

Three scenarios are presented in order to estimate the DC output Voltage and the efficiency depending on the channel characteristics: channel models of HyperLAN/2 (model AD-E), flat-frequency fading channel and frequency-selective fading channel.

The first case takes place with realistic simulated channels from a WLAN standard. Tab. II summarizes the channel models that were specified by this standard and used to perform the first simulation of this paper [9]. The channels are wideband, with Rayleigh or Rician modeled tapped delay lines. The environment in large office with line-of-sight (LOS) or nonline-of-sight (NLOS) propagation and large open-space NLOS are the most common channel environments of HyperLAN/2.

TABLE II

CHANNEL MODELS

\begin{tabular}{|c|c|c|c|c|}
\hline Model & $\begin{array}{c}\text { RMS } \\
\text { Delay } \\
\text { Spread }\end{array}$ & $\begin{array}{c}\text { Rician } \\
\text { Factor } \\
\text { First Tap }\end{array}$ & $\begin{array}{c}\text { Cha- } \\
\text { racteristic }\end{array}$ & Typical Environment \\
\hline A & $50 \mathrm{~ns}$ & - & Rayleigh & Office NLOS \\
\hline $\mathrm{D}$ & $140 \mathrm{~ns}$ & $10 \mathrm{~dB}$ & Rician & $\begin{array}{c}\text { Large } \\
\text { Open-space LOS }\end{array}$ \\
\hline $\mathrm{E}$ & $250 \mathrm{~ns}$ & - & Rayleigh & $\begin{array}{c}\text { Large } \\
\text { Open-space NLOS }\end{array}$ \\
\hline
\end{tabular}

For the second and third case, the input signal is faded by multiplying the fading coefficients generated using the Jakes's emulator tool from Keysights ADS software. These study cases take place in a situation of small-scale fading, so a large number of reflective paths is encountered and the received signal envelope is described by a Rayleigh or a Rician probability density function (PDF) [10]. The small-scale fading is assumed to be a frequency-flat fading or frequencyselective fading. The fading amplitudes can be modeled by a Rician or a Rayleigh distribution, depending on the presence or absence of specular signal component. Fading is Rayleigh if the multiple reflective paths are large in number and there is no dominant LOS propagation path. If there is still a dominant LOS path, then the fading is Rician-distributed. The fading amplitude $r_{i}$ at the $i_{t h}$ time instant can be represented as

$$
r_{i}=\sqrt{\left(x_{i}+\beta\right)^{2}+y_{i}^{2}}
$$

where $\beta$ is the amplitude of the specular component and $x_{i}, y_{i}$ are samples of zero-mean stationary Gaussian random processes each with variance $\sigma_{i}^{2}$. The ratio of specular to diffuse energy defines the so-called Rician K-factor, which is given by

$$
K=\frac{\beta^{2}}{2 \sigma_{o}^{2}}
$$




\section{RF Signal Source}

Signal source of the proposed WPT system is a multi-sine signal $x(t)$ and can be written as

$$
x(t)=\Re\left\{\sum_{n=0}^{N-1} S_{n} \cdot e^{j \theta_{n}} \cdot e^{j 2 \pi f_{n} t}\right\}
$$

where $S_{n}$ and $\theta_{n}$ refer to the amplitude and phase of the $n^{\text {th }}$ sine-wave at frequency $f_{n}$. The frequencies are evenly spaced with a frequency spacing $\Delta f=\mathrm{BW} / \mathrm{N}$, where $\mathrm{BW}$ is the bandwidth of the input signal, $\mathrm{BW}=20 \mathrm{MHz}$, and $\mathrm{N}$ the number of tones.

\section{Simulation Results}

\section{A. Multi-tone Performance}

In all following results, we have used a 4-tone source as a reference in order to establish a comparison (4-tone being also the reference scenario of [2]). Beforehand, we have used our simulation scheme without any channel modeling in order to verify the impact of using a multi-tone signal source.

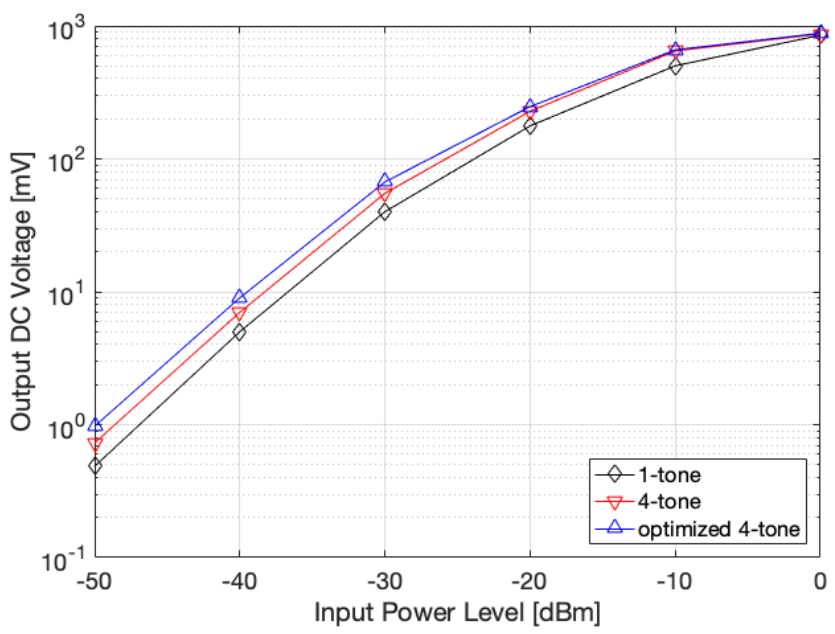

Fig. 2. DC voltage $(\mathrm{mV})$ as a function of the input power $(\mathrm{dBm})$ without channel.

As [2] shows, using a multi-sine signal can bring an interesting gain $(3.5 \mathrm{~dB}, 2.7 \mathrm{~dB}, 2.2$ at $-50 \mathrm{dBm},-30 \mathrm{dBm}$, $-10 \mathrm{dBm}$, respectively). However, in a simple case where only a 4 sine-wave is used as a multi-sine RF input source, without considering any channel at that point, the gain provided by the optimization of the amplitudes $S_{n}$ or phases $\theta_{n}$ remains low since it only relates to mitigate the nonlinear behavior of the Schottky diode $(2.5 \mathrm{~dB}, 1.7 \mathrm{~dB}, 0.1$ at $-50 \mathrm{dBm},-30$ $\mathrm{dBm},-10 \mathrm{dBm}$, respectively). We can now try to foresee if this optimization could offer better gains in more realistic channel conditions.

\section{B. Influence of The Channel}

The following simulations show the influence of the channel without bringing any optimization of the amplitudes $S_{n}$ or phases $\theta_{n}$, that is to say that the system only undergoes the channel conditions. Fig. 3 shows the output DC voltage as a function of the RF input power level for an optimized 4-tone without channel and with 3 specified channel models A, D and $\mathrm{E}$. The simulation shows the impact of the channel on the rectifier performance. Model D was chosen because this is the only Rician channel of HyperLAN/2 specification and model $\mathrm{A}$ and $\mathrm{E}$ were chosen because of the extreme values of the average RMS delay spread of the models of this standard, 50 ns and $250 \mathrm{~ns}$, respectively. With a R-C cell $15 \mathrm{k} \Omega / 20 \mathrm{pF}$ of the proposed rectifier, the constant time is $300 \mathrm{~ns}$. For model E, the highest values of average relative power are around the 18th tap, at $320 \mathrm{~ns}$. So, the values of the output DC voltage obtained are lower, below the case without propagation channel, the delay being greater than the time constant. For model A, the highest values of average relative power are found at the first tap, so for small values of delay, therefore the maximum of the input power level can be collected. As can be seen, a LOS model D or a NLOS model A situation bring a very low gain compared to a situation where the signal is transmitted without taking into account the channel (optimized 4-tone). But it is important to notice that at that point, no optimization is performed based on the channel conditions.

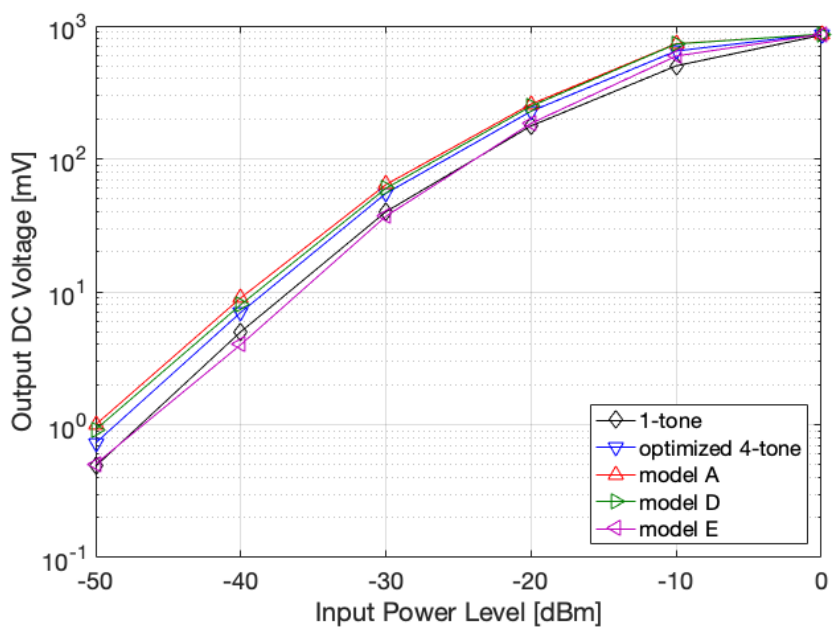

Fig. 3. DC voltage $(\mathrm{mV})$ as a function of the input power $(\mathrm{dBm})$ for specific channel models (A, D, E) of HyperLAN/2 standard.

Fig. 4 and Fig. 5 shows the output DC voltage as a function of the RF input power level for an optimized 4-tone source without channel, and with different values of the $\mathrm{K}$ constant of Rice's law. The simulation was performed in order to have a frequency-flat fading channel and a frequency-selective channel. The fading channel model block was set with 5 taps and having delays and average relative powers in order that the coherence bandwidth is larger than the bandwidth of the signal source $(20 \mathrm{MHz})$ for Fig. 4 and the opposite for Fig. 5. In the case of frequency-flat fading, the coherence bandwidth is $74 \mathrm{MHz}$ and in the case of frequency-selective fading, the coherence bandwidth is $9 \mathrm{MHz}$. For the two scenarios, the performance of the rectifier improves when $\mathrm{K}$ increases. As can be seen, there are more disparities in the case of frequency- 
flat fading where a factor of 10 appears between $\mathrm{K}=1$ and $\mathrm{K}$ $=100$ for low input power levels. However, in accordance with theoretical results from [4] and experimental results from [5], a frequency-flat fading channel brings less performance gain. On the other hand, being in an NLOS scenario with a very frequency-selective channel already brings a significant gain in performance whereas no optimization has yet been achieved.

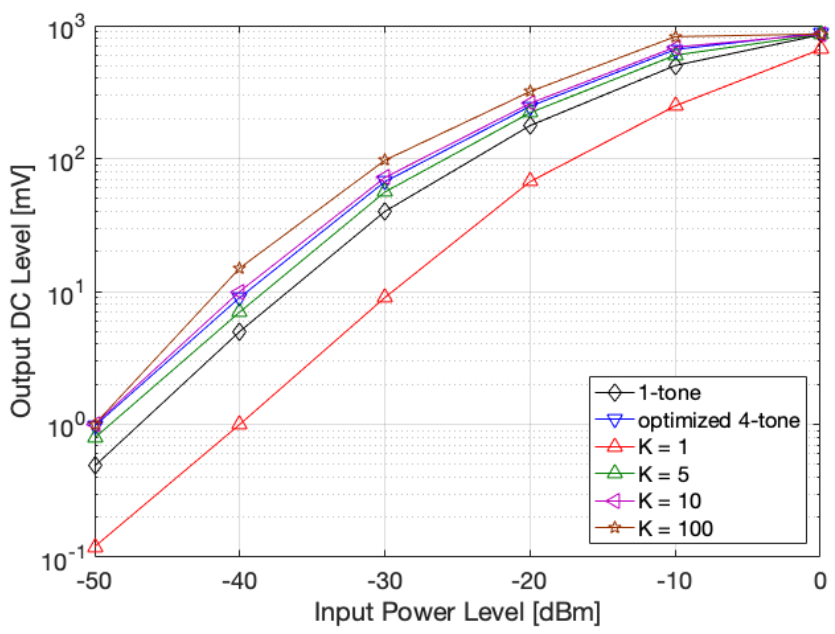

Fig. 4. DC voltage $(\mathrm{mV})$ as function of input power $(\mathrm{dBm})$ for a frequencyflat fading channel when $\mathrm{K}$ increases.

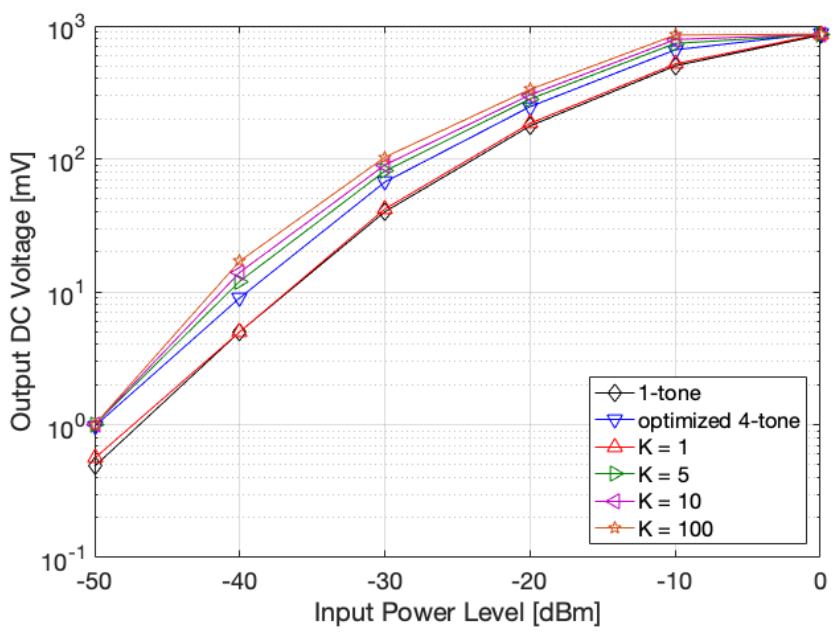

Fig. 5. DC voltage $(\mathrm{mV})$ as function of input power $(\mathrm{dBm})$ for a frequencyselective channel when $\mathrm{K}$ increases.

\section{Optimized Amplitudes and Phases with Realistic Channel}

Optimization tools of Keysights ADS software are used in order to adjust the amplitudes $S_{n}$ and phases $\theta_{n}$ of the multisine RF source in order to maximize the output DC voltage. In the case of a 4-tone, the optimization of these two parameters of the input RF signal is performed for an input power level of $-20 \mathrm{dBm}$ and for two values of the Rician factor $\mathrm{K}=5$ and $\mathrm{K}$ $=50$ of a frequency-selective channel. Simulation results are shown in Tab. III where $N_{a}$ denotes non-optimized amplitudes and $N_{b}$ denotes optimized amplitudes.

TABLE III

DC Collected Power Performance Results For -20 DBM

\begin{tabular}{|c|c|c|c|c|}
\hline & \multicolumn{2}{|c|}{$\mathrm{K}=5$} & \multicolumn{2}{c|}{$\mathrm{K}=50$} \\
\cline { 2 - 5 } & $N_{a}$ & $N_{b}$ & $N_{a}$ & $N_{b}$ \\
\hline DC Collected Power $(\mu \mathrm{W})$ & 4.06 & 5.3 & 4.06 & 7.17 \\
\hline Performance Gain $(\%)$ & 30.5 & & 76.6 \\
\hline
\end{tabular}

Taking into account fading channels and optimizing amplitudes of the RF multi-sine source give good results in terms of performance with improvements of $30.5 \%$ and $76.6 \%$.

\section{CONClusion}

A complete WPT system was designed in simulation with different models of propagation channel. The output DC voltage was compared between 4-tone non-adaptive amplitudes through fading channel and 4-tone with adaptive amplitudes. It is shown that taking into account the channel characteristics and a realistic modeling of the rectifier could offer high gains in the global efficiency. The forthcoming work is then to implement a complete state-space model system whose optimization algorithm decreases the processing time of optimized waveform in order to obtain the best performance of harvested DC power.

\section{ACKNOWLEDGMENT}

The authors thanks the support of the Spie ICS - Insa lyon research chair on IoT.

\section{REFERENCES}

[1] M. S. Trotter, J. D. Griffin and G. D. Durgin, "Power-Optimized Waveforms for Improving the Range and Reliability of RFID Systems", IEEE International Conference on RFID, Orlando, May 2009.

[2] A. S. Boaventura and N. B. Carvalho, "Maximizing DC Power in Energy Harvesting Circuits Using Multisine Excitation", IEEE MTT-S International Microwave Symposium, August 2011.

[3] F. Bolos, J. Blanco, A. Collado and A. Georgiadis, "RF Energy Harvesting From Multi-Tone and Digitally Modulated Signals", IEEE Transactions on Microwave Theory and Techniques, Volume 64, Issue 6, pp 1918-1927, May 2016.

[4] B. Clerckx and E. Bayguzina, "Waveform Design for Wireless Power Transfer", IEEE Transactions on Signal Processing, Volume 64, Issue 23, pp 6313-6328, August 2016.

[5] J. Kim, B. Clerckx and P. Mitcheson, "Prototyping and Experimentation of a Closed-Loop Wireless Power Transmission with Channel Acquisition and Waveform Optimization", IEEE Wireless Power Transfer Conference (WPTC), Taipei, June 2017.

[6] R. Rousseau, F. Hutu and G. Villemaud, "On the Use of Vector Fitting and State-Space Modeling to Maximize the DC Power Collected by a Wireless Power Transfer System," 2nd URSI Atlantic Radio Science Conference (URSI AT-RASC), Gran Canaria,, September 2018.

[7] M. Holters and U. Zözer, "A Generalized Method for the Derivation of Nonlinear State-Space Models from Circuit Schematics", IEEE European Signal Processing Conference, January 2015.

[8] Yong Huangl, Naoki Shinoharal and Hiroshi Toromura, "A Wideband Rectenna for 2.4 GHz-band RF Energy Harvesting", IEEE Wireless Power Transfer Conference (WPTC), June 2016.

[9] J. Medbo and P. Schramm, "Channel Models for HyperLAN/2", ETSI/BRAN document, No 3ER1085B, 1998.

[10] J. G. Proakis, "Digital Communications", 5th ed., McGraw-Hill, 2008. 